\title{
Notes on the problems of relativistic nuclear physics
}

\author{
S.M. Eliseev ${ }^{1}$ and O.S. Kosmachev ${ }^{1}$ \\ ${ }^{1}$ Joint institute for nuclear research, 141980, Dubna, Moscow Region, Russia
}

\begin{abstract}
This article presents some arguments in favor of the necessity and opportunity to describe leptons and quarks on the united relativistic basis. The necessity is caused by experimental results of the relativistic nuclear physics. The opportunity has become really attainable taking into account the structure of the lepton equation groups and all their substructures obtained during the latest years.
\end{abstract}

Recently elementary particle physics has faced two challenges: dark matter and matter under extreme conditions. The second of them originally appeared as a product of relativistic nuclear physics. Both of these new facts are not described by existing theoretical models. The reason is that here we meet new physics, not just new events in physics which can be obtained in the framework of the already known, established notions.

It may be stressed that in the description of the observable ("bright") matter there is a gap between the lepton and hadron sectors. Unitary symmetry is an essential component of the Standard Model (SM). Its success is related to the fact that it successfully referred hard fixed substructures to hadrons - the quarks. But SM has not received them from the general principles. The lepton sector fell out completely from this structural classification of hadrons. It also, like quarks, turned out to be structureless. CM assigns sets of individual properties to leptons and quarks in the form of quantum numbers, but it can not name the reasons of their origin. This situation contradicts to the principle of the cause-and-effect relationship.

This fact is reflected in the conclusions of the O'Raifertaigh theorem [1]: the unification of the Lorentz group and internal symmetries of hadrons in the framework of a single group is possible only in the form of a direct product. As a consequence, we get a conclusion: all the components of the hadron multiplet have equal masses - that is not true.

On the other hand, a holistic description of the lepton sector has been recently developed by us [2]-[5]. A number of strict mathematical facts has been found and among them are: the presence of the individual structure of each lepton equation and the analogue of the confinement. It is detected clearly in the structure of both stable and unstable equations and occurs without any ad hoc assumptions. This structural confinement is necessary to understand the presence of substructures in the composition of the observed particles which do not exist in free states. In this case they are maximum invariant subgroups on which the expressions for the currents are formed. These subgroups appear automatically, i.e. their existence has not been assumed in advance.

The variety of the structures of different lepton equations is the basis for describing various properties of the leptons observed in the experiment. It turns out that in the description of the leptons presented in the classical works by Dirac [6], Pauli [7] and Majorana [8], this requirement is fulfilled if the structure is understood as a group structure of each wave equation obtained in these three arti- 
cles. The group structures arise in the equations at the moment when you are writing them. However, until recently they have not been the subject of the study.

Indivisible structural components of all lepton equations are the four connectivity components of the Lorentz group. In this sense, relativism is the basis for the lepton equations. The four components of connectivity regulate their transformation properties relatively the discrete transformations: $(\mathrm{R})$ is spatial inversion; (T) is the change of the time direction; (RT) - is the combined effect of the two transformations. In the lepton sector we have got a quite clear evidence of the fundamentality of lepton equation grounds. They fit into five assumptions which are necessary and sufficient conditions to formulate the lepton equations. The full set of equations admissible by five assumptions includes the massive and massless, charged and neutral, stable and unstable leptons having spin of 1/2.

The above mentioned holistic description of the lepton sector differs from the situation observed in the hadron sector on the basis of the quark model. As already mentioned above the quarks have a set of quantum numbers (including electric charge) without substructures, which would act as carriers of the declared characteristics. The proclamation of the hard quark structure is not related to the requirements of relativity. Subsequent various ways of quark relativization differ from what can be observed in the lepton sector. This structure of the lepton equations is the consequence of the relativity requirements and not vice versa. Such a permutation in the above procedure, as the practice of the latest 50 years has shown, leads to the differences which are impossible to avoid [9].

However, it becomes clear that there are no contra-indications to compose structures of hadrons in the same algorithmic sequence, as for leptons [5]. But it is necessary for this goal to add some complementary symmetries. In this case, relativism will be the basis of hadron substructures, which play the role of quarks. Structural components of the equations for stable leptons are four connectivity components of the homogeneous Lorentz group in the form of infinitesimal operators. Each of the connectivity components in the group of stable lepton equations is a subgroup of the order of 16 . They are maximal subgroups which are the basis to form expressions for currents. We introduce the notations for them: group $\mathbf{d}_{\gamma}-$ is related with proper orthochronous representation; group $\mathbf{f}_{\gamma}-$ is related with the improper orthochronous or $(P)$ - conjugate representation; group $\mathbf{b}_{\gamma}-$ is related with the proper antichronous or $(T)$ - conjugate representation; group $\mathbf{c}_{\gamma}-$ is related with the antichronous improper or (PT)- conjugate representation.

The stable lepton groups have the following content:

the group of Dirac equation : $\mathbf{D}_{\gamma}(\mathbf{I I})=\left\{\mathbf{d}_{\gamma}, \mathbf{b}_{\gamma}, \mathbf{f}_{\gamma}\right\}$;

the group of Pauli equation for massless neutrinos: $\mathbf{D}_{\gamma}(\mathbf{I I I})=\left\{\mathbf{d}_{\gamma}, \mathbf{b}_{\gamma}, \mathbf{c}_{\gamma} \mathbf{f}_{\gamma}\right\}$;

the group of Majorana equation for massive neutrinos: $\mathbf{D}_{\gamma}(\mathbf{I})=\left\{\mathbf{d}_{\gamma}, \mathbf{c}_{\gamma}, \mathbf{f}_{\gamma}\right\}$.

By analogy with the Pauli equation group $\mathbf{D}_{\gamma}(\mathbf{I I I})$ it turned out to be possible to build additionally two groups with similar characteristics: $\mathbf{D}_{\gamma}(\mathbf{I V})$ and $\mathbf{D}_{\gamma}(\mathbf{V})$. Their peculiarity lies in the fact that each of them contains the connectivity components of one type only. In this case, they are candidates for massless particles, which have no antiparticles. However, it turns out that the both groups of the particles do not have the necessary elements to form the 4-vector current. Thus these particles can not exist in the vacuum (i.e. in the free states). Then it becomes clear that these groups appear automatically as substructures in groups of unstable leptons. Their structural content is the following: the group for the massless (T)-"singlet": $\mathbf{D}_{\gamma}(\mathbf{I V})=\left\{\mathbf{b}_{\gamma}\right\}$; the group for the massless (PT)-"singlet": $\mathbf{D}_{\gamma}(\mathbf{V})=\left\{\mathbf{c}_{\gamma}\right\}$.

For the unstable leptons the maximal invariant subgroups are subgroups of stable leptons which are part of each group of unstable leptons in various combinations.

The structures of the unstable lepton groups are as follows:

group $\boldsymbol{\Delta}_{\mathbf{1}}$ is related with $\mu$-lepton, $\boldsymbol{\Delta}_{\mathbf{1}}=\left\{\mathbf{D}_{\gamma}(\mathbf{I I}), \mathbf{D}_{\gamma}(\mathbf{I I I}), \mathbf{D}_{\gamma}(\mathbf{I V})\right\}$;

group $\Delta_{\mathbf{3}}$ is related with $\tau$-lepton, $\boldsymbol{\Delta}_{\mathbf{3}}=\left\{\mathbf{D}_{\gamma}(\mathbf{I I}), \mathbf{D}_{\gamma}(\mathbf{I}), \mathbf{D}_{\gamma}(\mathbf{I I I})\right\}$; 
group $\boldsymbol{\Delta}_{\mathbf{2}}$ is related with unstable neutrino, $\boldsymbol{\Delta}_{\mathbf{2}}=\left\{\mathbf{D}_{\gamma}(\mathbf{I}), \mathbf{D}_{\gamma}(\mathbf{I I I}), \mathbf{D}_{\gamma}(\mathbf{V})\right\}$.

Every group related with the corresponding equation has an original composition.

Some of the results of the holistic approach to describe the lepton sector are given below:

1. We have found an opportunity to list all types of lepton equations in the framework of the initial assumptions;

2. Structural individuality of each lepton equations has been determined;

3. The primary structural classification of the leptons has been obtained;

4. A simple and natural (without additional assumptions) opportunity has been found to describe the particles which do not exist in free states but occur in bounded states. It is the analogue of the confinement;

5. Basics of relativistic description of unstable leptons have been obtained. The structure of their equations indicates the internal self-consistency of the whole lepton sector in the framework of the proposed algorithm;

6. The existence of massive unstable neutrino has been predicted;

7. The existence of "doublets" of $\tau^{ \pm}$-leptons has been predicted, i.e. $\left(\tau^{*}\right)^{ \pm}$-leptons. These four leptons form a quartet state on the basis of a united group.

It is evident that taking into account the structure of the leptons leads to non-trivial consequences for the whole sector. It is clear that the analogous consequences for hadrons will arise in a greater quantity because of their more complex structure. Thus, in the lepton sector the relativism (or more exactly all four connectivity components of the Lorentz group) is the initial structure-forming factor. We can not say the same about quarks. They were postulated as the constituent elements of hadrons and then relativized. The consequent decades have not confirmed the equivalence of this sequence of actions in comparison with the Dirac algorithm. This is probably the case when the struggle with the consequences without considering the initial reasons does not lead to the success.

All unstable elementary particles finally decay into stable leptons and two non-lepton exceptions - photons and protons. This situation naturally raises questions on the possibility of the reverse processes associated with the formation of the observed particles on the basis mentioned above. A reasonable answer to this obvious question is not possible beyond the methods and opportunities of the relativistic nuclear physics. One of the consequences of solving this large scientific problem will be formation of the elementary particles kinetics.

The main sign of the formation of matter under extreme conditions is an unusual (exaggerated) multiplicity of secondary particles. Such processes are not conceivable outside the consistent relativity because the ratio $E=m c^{2}$ there has no alternatives. The initial phase of extreme states of matter (let's call it protoplasma) can not be in the equilibrium condition because further self-organization (i.e., processes which are inverse to decays) occurs in the result of formation and decays of intermediate different unstable particles. The Lagrange's method is not applicable in these nonholonomic systems and the models based on it become inefficient.

The relativistic nuclear physics clearly defined the importance of two processes that play a key role for the high multiplicity of secondary particles: maximum destructurization, i.e., the formation of the most simple up to indivisible components in the collision of relativistic nuclei and self-organization i.e. formation of the complex by means of more simple elements in the environment called the matter under extreme conditions. There is no sense to speak about complete destruction without considering 
the structure of colliding particles. The question for today is as follows: how complete and close to reality is our knowledge about the structure of nuclei, hadrons and leptons?

The option of the minimal components as a result of the complete destruction of the colliding nuclei becomes the first priority for understanding the matter under extreme conditions. Another option is how the observed particles are formed in this unusual environment.

The structures of the stable leptons are so simple that they can not decay in the vacuum. It follows that the structure of stable leptons is simpler than the structure of quarks. From the formulas of structural composition it is seen that the components of the leptons can not play a role of quarks. Therefore, the study of matter under extreme conditions puts forward the problem of hierarchy of the complexity. It is necessary to answer the following question - what is the content of the initial environment (protoplasma), which is equally suitable to form leptons and hadrons in the finite state as well as many of the resonances in the intermediate states, including baryonic clusters observed only in the collisions of relativistic nuclei [10].

Taking into account the situation, when the complex is formed on the basis of the simple, we can call the initial phase of matter under extreme conditions as photon-lepton protoplasma. It is clear that it does not exclude the formation and continued existence of other components including the type, which is now called the quark-gluon plasma.

Unstable particles can be considered as one of manifestations of the matter under extreme conditions limited by lifetime of the particle and finite spatial volume. Therefore, the completed and detailed study of their decays, taking into account their structure and the structure of decay products is the most simple and informative way for finding the self-organization puzzle. From this point of view a comprehensive study of the decays of unstable leptons seems to be promising at this stage.

In this case the confinement and deconfinement of particles to the above processes becomes significant for both vacuum and photon-lepton protoplasma. It becomes clear that the mechanism adopted today for confinement of quarks is not universal. It will require revision taking into account the influence of the lepton sector composition of matter in the extreme state. In particular, traditional confinement has no place in stable leptons, where substructures exist, but quarks do not. A similar situation is observed with unstable leptons.

Experimental relativistic nuclear physics has identified a number of problems, which will be updated and refined in the course of their study. The following problems are considered to be the first priority and play the key role:

1. It is necessary to find the description of leptons and quarks on the unified relativistic basis.

2. Search for the quark structure must be extended by using the Dirac's algorithm i.e., similarly how the structure of electrons and other leptons was understood. And taking into account additional symmetries beyond the minimum required for the lepton sector.

The difficulty and subtlety of the above is that it is necessary strongly to differentiate the properties of substructures and those characteristics which are the consequence of the interactions between the observed objects containing these substructures. The so-called quantum numbers are introduced only on the results of the interactions and then attributed to the particles in free states, and even to the particles which do not exist in the free states. The negative role of the above factors and difficulties will reduce while increasing the number of the described particles on the common basis. Changes in quark phenomenology are needed in order to gradually study the new challenges for it must be internally self-consistent and independent of unnecessary assumptions. 


\section{References}

[1] L. O’Raifertaigh, Phys. Rev. 139, B1052-B1062 (1964)

[2] A. A. Gusev, O. S. Kosmachev, Phys. Part. Nucl. Lett. 5, 67-71 (2008)

[3] O. Kosmachev, Phys. Part. Nucl. Lett. 7, 149-174 (2010)

[4] O. Kosmachev, Phys. of At. Nucl. 74(6), 930-935 (2011)

[5] O. Kosmachev, Phys. of At. Nucl. 75(7), 885-887 (2012)

[6] P. Dirac. Proc. Roy. Soc. A, 117, 610-624 (1928)

[7] W. Pauli, Handbuch der Physik (Springer, Berlin, 1933) 226

[8] E. Majorana, Il Nuovo Cimento 14, 171-184 (1937)

[9] A. Pais, Rev. Mod. Phys. 38(2), 215-255 (1966)

[10] A. M. Baldin et al., Phys. of Nucl. 52(5), 1427-1436 (1990) 